\title{
Original
}

\section{A Synergistic Antitumor Effect of Rituximab and Gamma Interferon Combined Therapy on Human CD20 + B-Cell Lymphoma Cells}

\author{
Shotaro KAmiJo ${ }^{1)}$, Hiromichi TsuchiYA ${ }^{* 2)}$, Toshihiko Gocho ${ }^{1)}$, \\ Yoshitaka YAMAZAKi $^{3)}$, Yui Akita ${ }^{4)}$, Yoko Ao $^{1)}$, \\ Akiko SASAKI $^{1)}$, Nana ICHIMURA ${ }^{1)}$ and Yuji KIUCHI ${ }^{1,2)}$
}

\begin{abstract}
Rituximab (RTX) is an anti-CD20 human-mouse chimeric monoclonal antibody that exhibits antibody-dependent, cell-mediated cytotoxicity and complementdependent cytotoxicity, resulting in an antitumor effect with immune cells or complement. RTX is approved for the treatment of many diseases including B cell lymphoma and rheumatoid arthritis. We examined whether combined RTX and gamma interferon (IFN $\gamma$ ) therapy provides a higher antitumor effect than RTX single therapy using B-cell lymphoma cells. In addition, we investigated the mechanisms underlying the antitumor effect. We treated tumor-derived cell lines with RTX alone, IFN $\gamma$ alone, or a combination of RTX and IFN $\gamma$ $(\mathrm{RTX}-\mathrm{IFN} \gamma)$. Untreated cells served as controls. We experimentally examined in vitro cell proliferation, conducted apoptosis and cell cycle assays, performed Western blotting to identify changes in the levels of proteins related to the cell cycle, and investigated tumor growth in a mouse xenograft experiment. Cell proliferation experiments indicated that RTX or IFN $\gamma$ alone did not significantly suppress cell growth compared with the control, whereas treatment with RTX-IFN $\gamma$ significantly suppressed cell proliferation. In vivo mouse experiments also showed that the administration of RTX-IFN $\gamma$ significantly suppressed tumor growth compared to the single therapies. Some tumors in mice treated with RTX-IFN $\gamma$ were completely resolved. The cell cycle assays revealed a significantly increased rate of cells in the G0/G1 phase following treatment with RTX-IFN $\gamma$ compared with the other groups, and the levels of $\mathrm{p} 27^{\mathrm{kip} 1}$ increased and the levels of cyclin E and Cdk 2 decreased in cells treated with RTX-IFN $\gamma$. Our findings suggested that RTX-IFN $\gamma$ combined therapy directly affects cells by arresting the cell cycle at the G1/S checkpoint and had a synergistic antitumor effect compared to RTX single treatment of B-cell lymphomas. This combined therapy may change the mortality rate for B-cell lymphomas.
\end{abstract}

Key words : rituximab, gamma interferon, combined therapy, cell cycle arrest

\footnotetext{
1) Department of Pharmacology, Showa University School of Medicine.

2) Department of Medical Education, Showa University School of Medicine, 1-5-8 Hatanodai, Shinagawa-ku, Tokyo 1428555, Japan.

3) Department of Hospital Phamacentics, Showa University Hospital.

4) Showa University School of Pharmacy.

* To whom corresponding should be addressed.
} 


\section{Introduction}

Rituximab (RTX) is an anti-CD20 human-mouse chimeric monoclonal antibody that shows antibody-dependent cell-mediated cytotoxicity (ADCC) and complement-dependent cytotoxicity (CDC). It is also the first monoclonal antibody to be used for clinical cancer therapy. RTX is combined with several chemotherapies for treating B cell lymphoma and is used alone to treat autoimmune diseases such as rheumatism. Clinical trials have already provided evidence on the safety of $\mathrm{RTX}^{1-4)}$.

Historically, it was anticipated that gamma interferon (IFN $\gamma$ ) would exhibit antitumor effects ; however, clinical trials in the 1980s on its efficacy as a cancer therapy showed no antitumor effects, thus IFN $\gamma$ was not pursued as a cancer therapy ${ }^{5)}$. IFN $\gamma$ is a type II interferon secreted as a cytokine by natural killer cells, dendritic cells, and CD8 T cells ${ }^{6-8)}$. Several reports indicate that selected molecular target drugs require type I and II interferon for efficacy ${ }^{9)}$.

We previously reported that combined anti-HER2 antibody and IFN $\gamma$ therapy showed a higher antitumor effect than anti-HER2 antibody single therapy ${ }^{10)}$. Her2 is regarded as an oncogene because overexpression of HER2 causes cells to become malignant. In contrast, CD20 is not an oncogene. In addition, cancers that overexpress HER2 form solid tumors, whereas lymphomas are humoral tumors.

In this study, we examined whether combined RTX and IFN $\gamma$ therapy (RTX-IFN $\gamma$ ) provides a higher antitumor effect than RTX single therapy using B-cell lymphoma cells. We also investigated the mechanisms related to any synergistic antitumor effects observed.

\section{Materials and methods}

\section{Cell lines and culture conditions}

The Raji and RAMOS cell lines are Burkitt's lymphoma cells that originated from B lymphocytes. Both cell lines were obtained from the American Type Culture Collection (ATCC, Manassas, VA) and were cultivated in RPMI-1640 growth medium containing L-glutamine and 10\% fetal bovine serum (Biosera, Kansas City, MO).

\section{Drugs}

RTX was obtained from Chugai (Chugai Pharmaceutical Co., Ltd., Tokyo, Japan) and human IFN $\gamma$ was obtained from PROSPEC (PROSPEC Protein Specialists, Rohovot, Israel). We diluted the RTX with phosphate-buffered saline (PBS) to a final concentration of $1 \mathrm{mg} / \mathrm{ml}$ and IFN $\gamma$ to a final concentration of $1 \times 10^{5} \mathrm{IU} / \mathrm{ml}$. For all RTX and IFN $\gamma$ combined treatment experiments, IFN $\gamma$ was added 30 minutes after the addition of RTX.

\section{Cell growth assay}

We used four groups of Raji and RAMOS cells : control group, RTX group, IFN $\gamma$ group, and RTX-IFN $\gamma$ group. Cells $\left(1 \times 10^{5}\right.$ of Raji or $5 \times 10^{5}$ of RAMOS $)$ were seeded onto 6-well plates ( $2 \mathrm{ml}$ of medium for Raji cells and $3 \mathrm{ml}$ of medium for RAMOS cells), one for each of the 
four groups, and cultured in RPMI-1640 containing L-glutamine and 10\% fetal bovine serum. Drug treatment was initiated 8 hours after the cell passage, and then the cells were cultured for a further 24, 48, or 72 hours. The cells were counted at each time using an automated cell counter (Bio-Rad Laboratories, Inc., Hercules, CA) and a cell growth curve was constructed. No drugs were added to the control group, the RTX group was treated with $10 \mu \mathrm{g} / \mathrm{ml}$ of RTX, the IFN $\gamma$ group was treated with $100 \mathrm{IU} / \mathrm{ml}$ of $\mathrm{IFN} \gamma$, and the RTX-IFN $\gamma$ group was treated with $10 \mu \mathrm{g} / \mathrm{ml}$ of RTX and $100 \mathrm{IU} / \mathrm{ml}$ of $\mathrm{IFN} \gamma$.

\section{Cell apoptosis assay}

Cells $\left(5 \times 10^{5}\right)$ were seeded onto 6-well plates, and then treated for 48 hours with RTX, IFN $\gamma$, or RTX-IFN $\gamma$; the control group was not treated with drugs. Following treatment, the cell number was adjusted to between $1 \times 10^{6}$ and $1 \times 10^{7} / 100 \mu$ in culture medium, and then stained using a Mouse Annexin V and Dead Cell Assay Kit ${ }^{\circledR}$ (Merck Group, Darmstadt, Germany). The cells were analyzed using a flow cytometer (Merck Group).

\section{Cell cycle analysis}

Cells $\left(1 \times 10^{5}\right)$ were seeded onto 6-well plates, then $2 \mathrm{mM}$ hydroxyurea was added to each well to synchronize the cell cycle phase for 12 hours. The cells were then treated for 8 hours with RTX, IFN $\gamma$, or RTX-IFN $\gamma$, or left untreated as control. The cells were then washed with PBS, collected by pipetting, and fixed with $75 \%$ cold ethanol for 4 hours at $-20^{\circ} \mathrm{C}$. The cells were incubated and the cell cycle phase was determined using a flow cytometer and a Cell Cycle Assay $\mathrm{Kit}^{\mathrm{R}}$ (Merck Group).

\section{Lysate preparation and Western blotting}

Cells $\left(1 \times 10^{6}\right)$ were seeded onto 6-well plates and cultured with each drug or the drug combination for 48 hours, and then lysed in RIPA buffer (Wako Pure Chemical Industries, Ltd., Osaka, Japan) containing a protease inhibitor cocktail (Sigma-Aldrich, St. Louis, MO), before analysis by Western blotting.

In brief, $40 \mu \mathrm{g}$ of protein per lane was separated by $12 \%$ SDS-polyacrylamide gel electrophoresis, and then electroblotted onto PVDF membranes (Bio-Rad Laboratories) for 1 hour in PBS containing 5\% nonfat milk (Wako Pure Chemical Industries, Ltd.) and 0.1\% Tween 20 (Wako Pure Chemical Industries, Ltd.). The membranes were incubated overnight at $4^{\circ} \mathrm{C}$ with a rabbit antibody against p27 $7^{\mathrm{kip} 1}$ (Cell Signaling Technology, Danvers, MA), a mouse antibody against cyclin E (Santa Cruz Biotechnology, Dallas, TX), or a mouse antibody against Cdk 2 (Santa Cruz Biotechnology). The membranes were then washed three times in PBS containing $0.1 \%$ Tween 20 and incubated for 1 hour with either a goat anti-rabbit IgG antibody conjugated with horseradish peroxidase (SeraCare Life Sciences, Milford, MA) as a secondary antibody for the $\mathrm{p} 27^{\mathrm{kip} 1}$ primary antibody or a goat anti-mouse $\mathrm{IgG}$ antibody conjugated with horseradish peroxidase (Santa Cruz Biotechnology, Dallas, TX) as a secondary antibody for the cyclin E and Cdk 2 primary antibodies. Protein bands were measured using a LI-COR $3600^{\circledR}$ 
computerized digital imaging system (LI-COR, Inc., Lincoln, NE) .

\section{Mouse in vivo experiment}

Eight-week-old female C.B-17 SCID mice, which have a severe combined immunodeficiency affecting both $\mathrm{B}$ and $\mathrm{T}$ lymphocytes, were purchased (CHARLES RIVER LABORATORIES JAPAN, Inc., Yokohama, Japan) and bred for one week to allow adjustment prior to the experiment. Raji cells $\left(1 \times 10^{7}\right)$ were injected subcutaneously into both sides of the back of each mouse.

We divided the mice into four groups so that the average tumor size in each group was similar and initiated drug treatment 14 days after cell inoculation. The control group was treated with $100 \mu \mathrm{l}$ PBS, the RTX group with $100 \mathrm{mg} / 100 \mu \mathrm{RTX}$ in PBS, the IFN $\gamma$ group with $10,000 \mathrm{IU} / 100 \mu \mathrm{IFN} \gamma$ in PBS, and the RTX-IFN $\gamma$ combined therapy group with both drugs. The drugs were delivered by intraperitoneal injection. For the combined therapy group, IFN $\gamma$ was injected 30 minutes after the RTX injection. The experiment was performed for four weeks. Tumors were measured three times a week using a digital caliper and tumor size was calculated as length $\times$ width $\times$ height. The drugs were administered three times a week.

\section{Statistical analysis}

Statistical analysis was carried out using YSAT 2013 (Igakutosho-shuppan Ltd., Toda, Japan). Differences in mean values were statistically analyzed using non-repeated measures ANOVA, followed by Dunnett's test or the Student-Newman-Keuls test. Tumor volume differences between the RTX and RTX-IFN $\gamma$ groups were statistically analyzed using unpaired Student's t-test. The threshold for significance was $P<0.05$ in all statistical analyses.

\section{Results}

\section{Cell proliferation}

- Raji cells

RTX-IFN $\gamma$ treatment was more effective at suppressing cell proliferation than RTX treatment on Day 2 and Day 3 after drug treatment. On Day 3, proliferation of the RTX-IFN $\gamma$ group cells was $60 \%$ of the control group, whereas that of the RTX group was $89 \%$ of the control group. Cell counts were significantly decreased in the RTX-IFN $\gamma$ treatment group compared to the control group, whereas the RTX and IFN $\gamma$ groups showed no significant difference compared to the control group. Indeed, the IFN $\gamma$ group showed an increased number of Raji cells (Fig. 1a).

- Ramos cells

The same in vitro experiment using RAMOS cells showed that cell proliferation in the RTXIFN $\gamma$ group was $69 \%$ of the control group on Day 3, whereas proliferation of the RTX group was $88 \%$ of the control group on Day 3. Also on Day 3, the RTX-IFN $\gamma$ group showed significant suppression of cell proliferation compared to the control group, whereas treatment with RTX or IFN $\gamma$ alone showed no significant difference compared to the control group (Fig. 1b). 
a

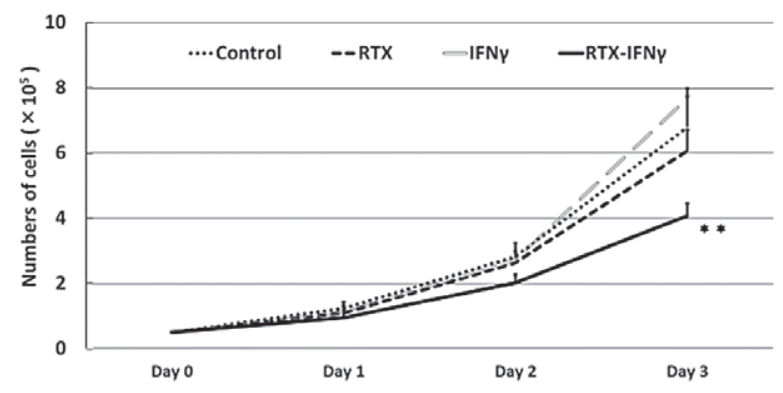

b

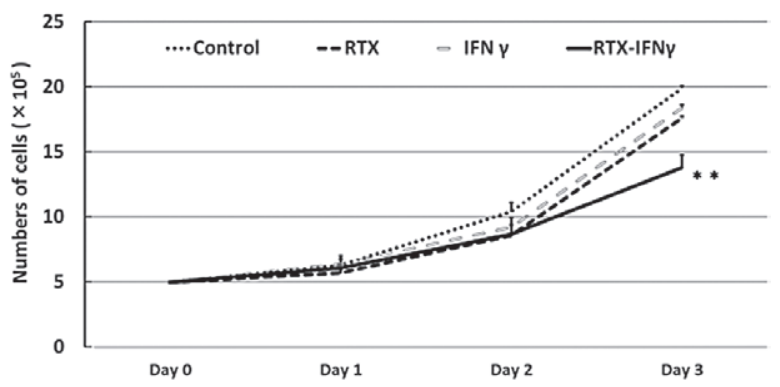

Fig. 1. B cell lymphoma cell proliferation after treatment with RTX, IFN $\gamma$, or RTX-IFN $\gamma$

a : Raji cell proliferation

Cells were divided into four groups of $1 \times 10^{5}$ cells : control, RTX $(10 \mu \mathrm{g} / \mathrm{ml})$, IFN $\gamma$ (100 IU/ml), and RTX-IFN $\gamma(10 \mu \mathrm{g} / \mathrm{ml}+100 \mathrm{IU} / \mathrm{ml}$; IFN $\gamma$ was added 30 minutes after RTX addition). Drug treatment was started 8 hours after passage, and cells were counted at 24, 48, and 72 hours. The curves show the mean + standard deviation of the number of cells from independent 3 culture experiments. Treatment with RTX resulted in no significant decrease compared to the control, whereas treatment with RTX-IFN $\gamma$ caused a significant difference compared to the control on Day 3 (Dunnett's test, ${ }^{* *} P<0.01$ vs. control, $\mathrm{N}=3$ ).

$\mathrm{b}$ : RAMOS cell proliferation

Cells $\left(5 \times 10^{5}\right)$ were seeded in 6-well plates with $3 \mathrm{ml}$ of medium and divided into four groups: control, RTX $(10 \mu \mathrm{g} / \mathrm{ml})$, IFN $\gamma(100 \mathrm{IU} / \mathrm{ml})$, and RTX-IFN $\gamma(10 \mu \mathrm{g} / \mathrm{ml}+100 \mathrm{IU} / \mathrm{ml}$; IFN $\gamma$ was added 30 minutes after the additions of RTX). Drug treatment was started 8 hours after passage and the cells were counted at 24, 48, and 72 hours. The curves show the mean + standard deviation of the number of cells from three independent culture experiments. Treatment with RTXIFN $\gamma$ significantly suppressed cell proliferation compared with the control, whereas treatment with RTX or IFN $\gamma$ alone resulted in no significant difference in cell proliferation compared to the control on Day 3 (Dunnett's test, ${ }^{* *} P<0.01$ vs. control, $\mathrm{N}=3$ ).

\section{Cell apoptosis assay}

The percentage of apoptotic cells was not significantly different among the four groups, as shown by flow cytometry (Fig. 2).

\section{Cell cycle assay}

We subjected Raji cells to a cell cycle assay to investigate why RTX-IFN $\gamma$ treatment suppressed cell proliferation without affecting apoptosis.

The percentage of cells in G0/G1 phase was significantly increased in the RTX-IFN $\gamma$ group compared with that in the other three groups (Fig. 3), and there was no significant difference in the percentage of cells in S phase and G2/M phase among the four groups (data not shown).

Cells treated with RTX or IFN $\gamma$ alone showed no significant difference in the percentage of cells in G0/1 phase compared with the control group, and RTX induced G1/S checkpoint cell arrest only when combined with IFN $\gamma$.

\section{Western blotting}

We then investigated the cell arrest findings at the G1/S checkpoint in cells treated with RTXIFN $\gamma$ using Western blot analysis. The expression level of $\mathrm{p} 27^{\mathrm{kip} 1}$ protein was increased and 


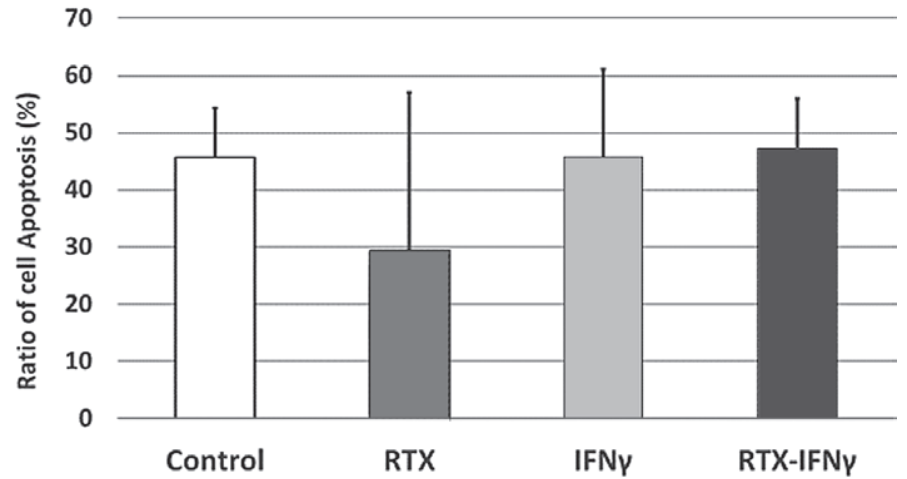

Fig. 2. Percentage of apoptotic cells after treatment with RTX, IFN $\gamma$, or RTX-IFN $\gamma$

We evaluated the difference in the number of apoptotic cells using flow cytometry based on propidium iodide and Annexin $\mathrm{V}$ staining after 48 hour incubation with RTX, IFN $\gamma$, or RTX-IFN $\gamma$. Cells $\left(5 \times 10^{5}\right)$ were seeded in 6 -well plates. The cells were divided into four groups: control, RTX $(10 \mu \mathrm{g} / \mathrm{ml})$, IFN $\gamma \quad(100 \mathrm{IU} / \mathrm{ml})$, and RTX-IFN $\gamma(10 \mu \mathrm{g} / \mathrm{ml}+100 \mathrm{IU} / \mathrm{ml})$. The figure shows the mean + standard deviation of the ratio of apoptotic Raji cells from three independent culture experiments. The data excluded debris.

There was no significant difference among the four groups in the ratio between apoptotic and living cells $(\mathrm{N}=3)$.

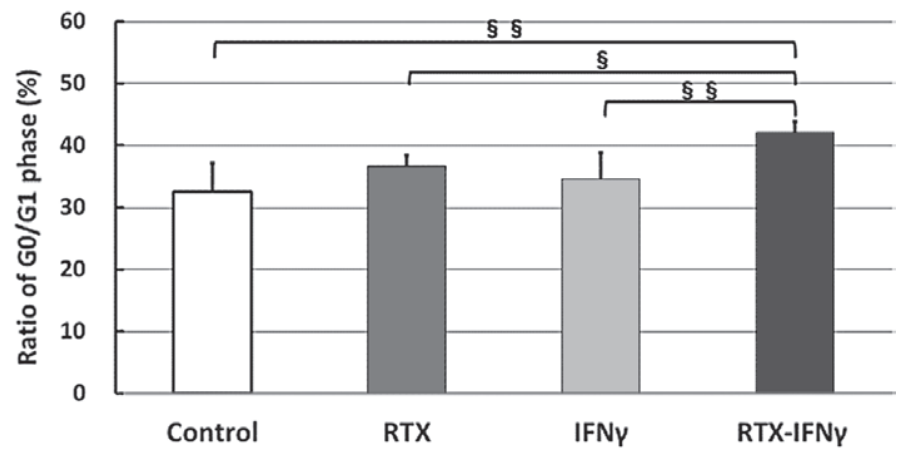

Fig. 3. Ratio of the cell number in the G0/G1 phase of the cell cycle

Raji cells were examined using a cell cycle assay. Cells $\left(5 \times 10^{5}\right)$ were seeded in 6-well plates and either left untreated (control) or were treated with RTX, IFN $\gamma$, or RTX-IFN $\gamma$ for 48 hours. The DNA of the cells was stained and the cells were examined using a flow cytometer. The figure shows the mean + standard deviation of the ratio of Raji cells in the G0/G1cell cycle phase from independent culture experiments; numbers of experiments shown below (Cont : $\mathrm{N}=4$, RTX: $\mathrm{N}=6$, $\mathrm{IFN} \gamma: \mathrm{N}=5, \mathrm{RTX}+\mathrm{IFN} \gamma: \mathrm{N}=6)$.

The percentage of cells in the G0/G1 phase significantly increased in the RTX-IFN $\gamma$ group compared to the other groups, whereas there was no significant difference among the control, RTX, and IFN $\gamma$ groups (Student-Newman-Keuls test; $\S P<0.05$ and $\S \S P<0.01$ ).

that of both Cdk 2 and Cyclin E was decreased in the RTX-IFN $\gamma$ group in the Raji cell line compared to the other groups (Fig. 4).

\section{Antitumor effect in vivo}

We conducted in vivo experiments in mice to investigate the antitumor effect of RTX-IFN $\gamma$ administration and the effect of ADCC and CDC. 
RTX and RTX-IFN $\gamma$ significantly suppressed tumor volumes in mice compared to the control after Day 9. Treatment with IFN $\gamma$ alone showed no antitumor effect in the mouse xenograft model (Fig. 5).

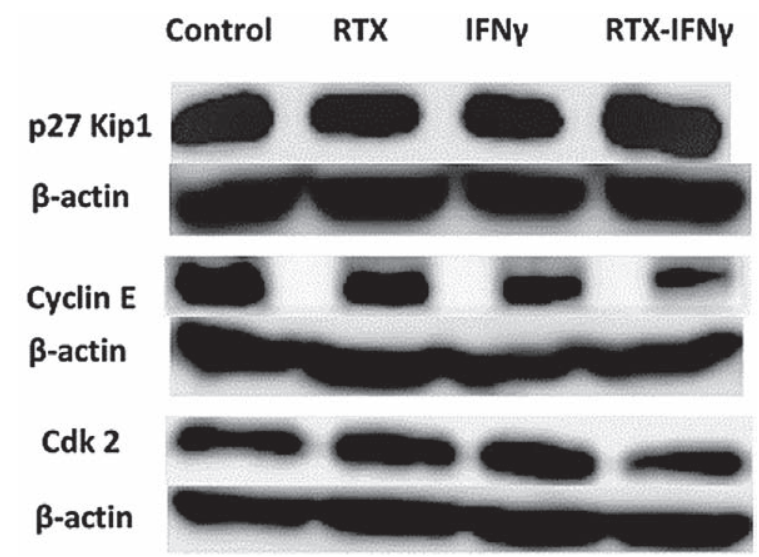

Fig. 4. Western blot analysis of G1/S checkpoint-related proteins

We investigated the protein expression levels of $\mathrm{p} 27^{\mathrm{kip} 1}$, cyclin $\mathrm{E}$, and $\mathrm{Cdk} 2$, which are related to G1/S checkpoint arrest, using Western blotting. Raji cells $\left(1 \times 10^{6}\right)$ were seeded in 6-well plates and cultured with each drug for 48 hours.

The expression level of $\mathrm{p} 27^{\mathrm{kip} 1}$ increased and that of cyclin E and Cdk 2 decreased in the RTX-IFN $\gamma$ group compared to the other groups.

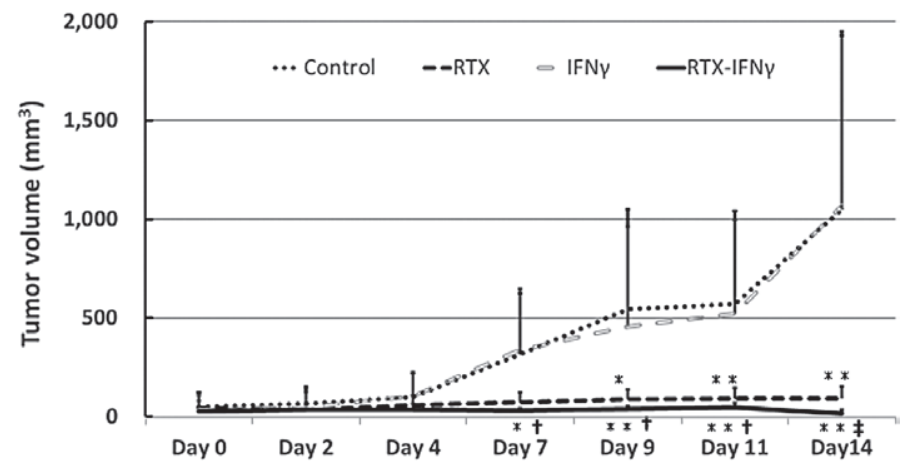

Fig. 5. Antitumor effect of RTX-IFN $\gamma$ in the C.B-17 SCID mouse xenograft model

C.B-17 SCID mice were used. Raji cells $\left(1 \times 10^{7}\right)$ were injected subcutaneously into both sides of the back of each mouse.

We divided the mice into 4 groups: control, PBS $100 \mu \mathrm{l}$; RTX $100 \mathrm{mg} / 100 \mu \mathrm{l}$ PBS; IFN $\gamma$ $100 \mathrm{IU} / 100 \mu \mathrm{l}$ PBS; RTX-IFN $\gamma(100 \mathrm{mg} / 100 \mu \mathrm{l} \mathrm{PBS}+100 \mathrm{IU} / 100 \mu \mathrm{l} \mathrm{PBS})$ and started drug treatment at Day 14 post-injection (Day 0 in the figure).

Tumor volumes were calculated as length $\times$ width $\times$ height. Measurements and drug treatments were performed three times a week. The curve shows the mean + standard deviation of the volume of the tumors; the numbers of tumors shown as below (Cont: $\mathrm{N}=4$, RTX: $\mathrm{N}=6$, IFN $\gamma: \mathrm{N}=5$, RTXIFN $\gamma: \mathrm{N}=6$ ).

Treatment with RTX and RTX-IFN $\gamma$ significantly suppressed tumor volumes compared with the control after Day 9. Mice treated with $\mathrm{RTX}-\mathrm{IFN} \gamma$ showed complete tumor resolution by Day 14, whereas treatment with RTX alone caused tumor regression. RTX-IFN $\gamma$ significantly decreased tumor volumes compared with RTX alone after Day 7. (Dunnett's test; ${ }^{*} P<0.05$ and ${ }^{* *} P<0.01$ vs. control, unpaired Student's t-test $\dagger P<0.05$ and $\$ P<0.01$ vs. RTX alone). 


\section{Discussion}

Tumor-derived cells were treated with RTX alone, IFN $\gamma$ alone, or RTX-IFN $\gamma$. Untreated cells served as controls. We experimentally examined in vitro cell proliferation, conducted apoptosis and cell cycle assays, performed Western blotting to identify changes in the levels of proteins related to the cell cycle, and investigated tumor growth in a mice xenograft experiment.

In cell proliferation experiments, treatment with RTX or IFN $\gamma$ alone induced no significant suppression compared with the control, whereas treatment with RTX-IFN $\gamma$ significantly suppressed cell proliferation. In the in vivo mouse experiments, the administration of RTX-IFN $\gamma$ significantly suppressed tumor growth compared with the single therapies. Cell cycle assays showed that the ratio of cells in G0/G1 phase significantly increased following exposure to RTX-IFN $\gamma$ compared with the other groups. Furthermore, RTX-IFN $\gamma$ treatment increased the level of p27 $7^{\text {kip } 1}$ protein and decreased the levels of Cyclin $\mathrm{E}$ and $\mathrm{Cdk} 2$ protein in the single treatment groups.

\section{Treatment of lymphomas with RTX}

RTX is an anti-human CD20 antibody used to treat various diseases because normal B cells express CD20 on the surface from the Pro B cell phase. B-cell non-Hodgkin lymphoma is treated by administering RTX combined with chemotherapeutic agents such as cyclophosphamide, hydroxydaunorubicin, oncovin and prednisolone $(\mathrm{CHOP})$ is used as a first-line treatment. RTX can enhance the antitumor effect of chemotherapeutics and decrease the required drug dose and associated side effects. Therefore, RTX may allow the safer use of chemotherapeutics by patients unable to tolerate full-dose chemotherapy.

\section{A new mechanism for an antitumor effect: arresting the cell cycle}

IFN $\gamma$ alone showed no antitumor effect in either in vitro or in vivo experiments, whereas treatment with RTX-IFN $\gamma$ showed a higher antitumor effect than RTX alone. This is consistent with a previous study showing that trastuzumab and IFN $\gamma$ combined therapy extended tumorfree survival ${ }^{10)}$. Indeed, some tumors in the present study were completely resolved following treatment with RTX-IFN $\gamma$. RTX-IFN $\gamma$ combined therapy may therefore strongly enhance the antitumor effects of clinical chemotherapeutics, allowing their dose to be reduced. Therefore, treatment with RTX-IFN $\gamma$ might extend the survival rate of patients with B cell lymphoma.

RTX is thought to exhibit ADCC and CDC, resulting in an antitumor effect; however, $\mathrm{ADCC}$ and $\mathrm{CDC}$ require secondary signals. In the presented in vitro experiments, the medium lacked effector cells or active complement and RTX single therapy did not significantly reduce cell numbers compared with the control. In contrast, treatment with RTX-IFN $\gamma$ reduced cell numbers in the absence of effector cells or active complement in the presented in vitro experiments.

Trastuzumab can induce antibody internalization within 24 hours after binding to erbB2 receptors on the cell membrane, resulting in apoptosis ${ }^{11)}$. Trastuzumab also can induce signal transduction and phenotypic changes in cells. In contrast, RTX does not induce antibody- 
internalization and thus cannot induce cell apoptosis ${ }^{12)}$. We observed no significant differences in apoptosis among the four groups in the current study, thereby demonstrating that treatment with RTX-IFN $\gamma$ has an antitumor effect without causing apoptosis and necrosis.

\section{Suppressing the malignant potential of cancer by arresting the cell cycle}

There are several reports that treatment of HER2-positive breast cancer with trastuzumab can change the malignant phenotype ${ }^{13-15)}$. The cell cycle assay used in this study showed a significant increase in the number of cells in the G0/G1 phase following treatment with RTX-IFN $\gamma$ compared with the other three groups. We therefore investigated several proteins related to the G1/S checkpoint using Western blotting and found that p27 ${ }^{\mathrm{kip} 1}$ increased and cyclin $\mathrm{E}$ and Cdk 2 decreased in cells treated with $\mathrm{RTX}-\mathrm{IFN} \gamma$. These changes might reflect cell cycle arrest at the G1/S checkpoint and suggest that RTX-IFN $\gamma$ can change the malignant phenotype to arrest the cell cycle.

Cells undergoing abnormal cell proliferation tend to accumulate genetic mutations ${ }^{16-19)}$, which can in turn increase the malignant potential of cancers and remarkably increase patient mortality, because the mutations promote adaptation to the microenvironment, metastatic ability, and treatment resistance ${ }^{20-22)}$. RTX-IFN $\gamma$ can affect the G1/S checkpoint and arrest the cell cycle leading to suppression of mutagenesis, thereby preventing the accumulation of gene mutation and subsequent enhancement of carcinogenesis.

\section{$R T X$ treatment for autoimmune diseases}

RTX reduces the number of plasma cells with ADCC and CDC, and is used for the treatment of many diseases such as idiopathic thrombocytopenic purpura, rheumatoid arthritis, and systemic lupus erythematosus ${ }^{23)}$. In this study, we demonstrated that RTX-IFN $\gamma$ reduced the number of B-cells, suggesting that treatment with RTX-IFN $\gamma$ could be more effective for the treatment of these diseases compared to RTX alone.

\section{Limitations}

We did not investigate the antitumor effect of RTX-IFN $\gamma$ combined with chemotherapies.

We also did not investigate markers related to the epithelial-mesenchymal transition, such as Snail, Slug, and TWIST, or the relationship between cell cycle arrest and epithelial-mesenchymal transition by measuring TGF- $\beta$.

\section{Conclusions}

RTX-IFN $\gamma$ combined therapy may induce a synergistic antitumor effect superior to RTX single therapy. RTX-IFN $\gamma$ acts via a new antitumor mechanism, affecting cancer cells by arresting the cell cycle. Our study suggests that RTX-IFN $\gamma$ treatment may enhance clinical chemotherapies, reduce the dose of chemotherapeutics, and extend tumor-free survival. RTX-IFN $\gamma$ is expected to arrest the cell cycle and therefore prevent increase in malignancy. 


\section{Conflict of interest disclosure}

The authors have no conflict of interest to declare.

\section{References}

1) Bose P, Gandhi V. Recent therapeutic advances in chronic lymphocytic leukemia. F1000Res. 2017;6:1924. (accesed 2018 Jan 10) Available from: https://f1000research.com/articles/6-1924/v1

2) Tudesq JJ, Cartron G, Riviere S, et al. Clinical and microbiological characteristics of the infections in patients treated with rituximab for autoimmune and/or malignant hematological disorders. Autoimmun Rev. 2018;17:115-124.

3) Schioppo T, Ingegnoli F. Current perspective on rituximab in rheumatic diseases. Drug Des Devel Ther. 2017;11:2891-2904.

4) Davies A, Berge C, Boehnke A. Subcutaneous rituximab for the treatment of B-cell hematologic malignancies: a review of the scientific rationale and clinical development. Adv Ther. 2017;34:2210-2231.

5) Miller C, Maher S, Young H. Clinical use of interferon-gamma. Ann N Y Acad Sci. 2009;1182:69-79.

6) Schroder K, Hertzog PJ, Ravasi T, et al. Interferon-gamma: an overview of signals, mechanisms and functions. $J$ Leukoc Biol. 2004;75:163-189.

7) Boehm U, Klamp T, Groot M, et al. Cellular responses to interferon-gamma. Annu Rev Immunol. 1997;15:749-795.

8) $\mathrm{Hu} \mathrm{X}$, Ivashkiv LB. Cross-regulation of signaling pathways by interferon-gamma: implications for immune responses and autoimmune diseases. Immunity. 2009;31:539-550.

9) Stagg J, Loi S, Divisekera U, et al. Anti-ErbB-2 mAb therapy requires type I and II interferons and synergizes with anti-PD-1 or anti-CD137 mAb therapy. Proc Natl Acad Sci USA. 2011;108:7142-7147.

10) Nagai Y, Tsuchiya H, Runkle EA, et al. Disabling of the erbB pathway followed by IFN-gamma modifies phenotype and enhances genotoxic eradication of breast tumors. Cell Rep. 2015;12:2049-2059. Erratum: in Cell Rep. 2015;13:450.

11) Baldassarre T, Truesdell P, Craig AW. Endophilin A2 promotes HER2 internalization and sensitivity to trastuzumab-based therapy in HER2-positive breast cancers. Breast Cancer Res. 2017;19:110. (accessed 2018 Jan 10 ) Available from: https://www.ncbi.nlm.nih.gov/pmc/articles/PMC5627411/

12) Shan D, Ledbetter JA, Press OW. Apoptosis of malignant human B cells by ligation of CD20 with monoclonal antibodies. Blood. 1998;91:1644-1652.

13) Vogel CL, Cobleigh MA, Tripathy D, et al. Efficacy and safety of trastuzumab as a single agent in first-line treatment of HER2-overexpressing metastatic breast cancer. J Clin Oncol. 2002;20:719-726.

14) Slamon DJ, Leyland-Jones B, Shak S, et al. Use of chemotherapy plus a monoclonal antibody against HER2 for metastatic breast cancer that overexpresses HER2. N Engl J Med. 2001;344:783-792.

15) Connell CM, Doherty GJ. Activating HER2 mutations as emerging targets in multiple solid cancers. ESMO Open. 2017;2:e000279. (accessed 2018 Jan 10) Available from: https:/www.ncbi.nlm.nih.gov/pmc/articles/PMC5708307/

16) Maes A, Menu E, Veirman K, et al. The therapeutic potential of cell cycle targeting in multiple myeloma. Oncotarget. 2017;8:90501-90520.

17) Ouderkirk-Pecone JL, Goreczny GJ, Chase SE, et al. Myosin 1e promotes breast cancer malignancy by enhancing tumor cell proliferation and stimulating tumor cell de-differentiation. Oncotarget. 2016;7:46419-46432.

18) Huang F, Chen J, Wang Z, et al. delta-Catenin promotes tumorigenesis and metastasis of lung adenocarcinoma. Oncol Rep. 2018;39:809-817.

19) Senft D, Qi J, Ronai ZA. Ubiquitin ligases in oncogenic transformation and cancer therapy. Nat Rev Cancer. 2018;18:69-88.

20) Zhang K, Yuan Q. Current mechanism of acquired resistance to epidermal growth factor receptor-tyrosine kinase inhibitors and updated therapy strategies in human nonsmall cell lung cancer. J Cancer Res Ther. 2016;12 


\section{(Supplement):C131-C137.}

21) Kuczynski EA, Sargent DJ, Grothey A, et al. Drug rechallenge and treatment beyond progression: implications for drug resistance. Nat Rev Clin Oncol. 2013;10:571-587.

22) Otsuki Y, Saya H, Arima Y. Prospects for new lung cancer treatments that target EMT signaling. Dev Dyn. 2018;247:462-472.

23) Lunardon L, Payne AS. Rituximab for autoimmune blistering diseases: recent studies, new insights. G Ital Dermatol Venereol. 2012;147:269-276.

[Received January 16, 2018 : Accepted January 19, 2018] 